\title{
ТРАНСПОРТНАЯ СЕТЬ 5G/IMT-2020
}

Е.Богданова, инженер компании "T8" / bogdanova@t8.ru

УДК 621.39, DOI: 10.22184/2070-8963.2019.84.7.40.47

Рассмотрены основные тенденции, которые будут актуальны для разработки линейки транспортного оборудования для мобильных систем пятого поколения IMT-2020/5G; описана архитектура решения в плоскости как передачи данных, так и управления и администрирования. Отмечается, что модернизация транспортной инфраструктуры неизбежна ввиду новых требований со стороны оборудования радиодоступа.

В июле 2017 года распоряжением Правительства России утверждена программа "Цифровая экономика Российской Федерации", одним из направлений которой является развитие технологий беспроводной связи. Необходимость развития мобильной сети обусловлена непрерывным ростом трафика и сопутствующей тенденцией увеличения пропускной способности. Кроме того, новые типы сервисов диктуют требования ко всему комплексу оборудования 5С в части задержек, надежности, инфраструктуры.

Международная организация ЗСРP (англ. 3СРP - 3 Generation Partnership Project) выделяет следующие сценарии применения технологии 5C/IMT2020 (англ. 5C/IMT-2020 - $5^{\text {th }}$ Generation International Mobile Telecommunications - 2020): сверхширокополосный мобильный доступ (англ. еMBB - Enhanced Mobile Broadband), сверхнадежные соединения с низкими задержками (англ. URLLC - Ultra-Reliable Low Latency Communication), повсеместные межмашинные соединения (mMTC - Massive Machine Type Communication).

Экосистема 5С состоит из трех частей: сети радиодоступа, транспортной сети и опорной сети. Каждая из составляющих - это отдельное направление разработки и целый стек технологий разного уровня. На сегодняшний день партнерство 3СРР утвердило первую фазу стандарта 5C/IMT-2020 - релиз 15. Выпуск 16-го релиза планируется в декабре 2019 года. Существующая версия содержит общее описание абонентских и базовых станций, радиоинтерфейсов и протоколов, архитектуру сети радиодоступа, однако практически не затрагивает построение транспортной сети. В то же время необходимая пропускная способность, задержки и инфраструктурная доступность во многом определяются именно этим компонентом.
По результатам опроса 72-х крупнейших в мире операторов связи, наиболее важной транспортной технологией признается стандарт OTN (англ. OTN - Optical Transport Network), неразрывно связанный с технологией спектрального уплотнения DWDM (англ. DWDM - Dense Wavelength Division Multiplexing), а также Ethernet в различных его модификациях (TSN Ethernet, Flex Ethernet, 25C Ethernet) [1].

В данной статье будут рассмотрены основные требования к транспортному оборудованию и технологии, которые могут обеспечить их выполнение, а также механизмы адаптации привычных стандартизированных решений к новым вызовам в 5С.

\section{АРХИТЕКТУРА ТРАНСПОРТНОЙ СЕТИ IMT-2020/5G}

В материалах 3СРP, а также 15-й исследовательской комиссии телекоммуникационного отделения Международного союза электросвязи (МСЭ-Т) выделено три транспортных сегмента: Fronthaul, Middlehaul, Backhaul, что в первом приближении схоже с уровнем доступа, метросегментом и магистральным участком традиционной сети связи (рис.1).

Особый интерес для производителей транспортного оборудования представляет Fronthaul, связывающий удаленные радиомодули RRU и распределенные модули gNB-DU, как правило, оптической волоконной сетью. Выделение этого сегмента характерно именно для пятого поколения и объясняется тенденцией к централизованной радиообработке с простыми модулями RRU, выполняющими первичные преобразования физического уровня в макрои микросотах, и сложными real-time и non-real-time алгоритмами ближе к ядру сети. Наиболее строгие 


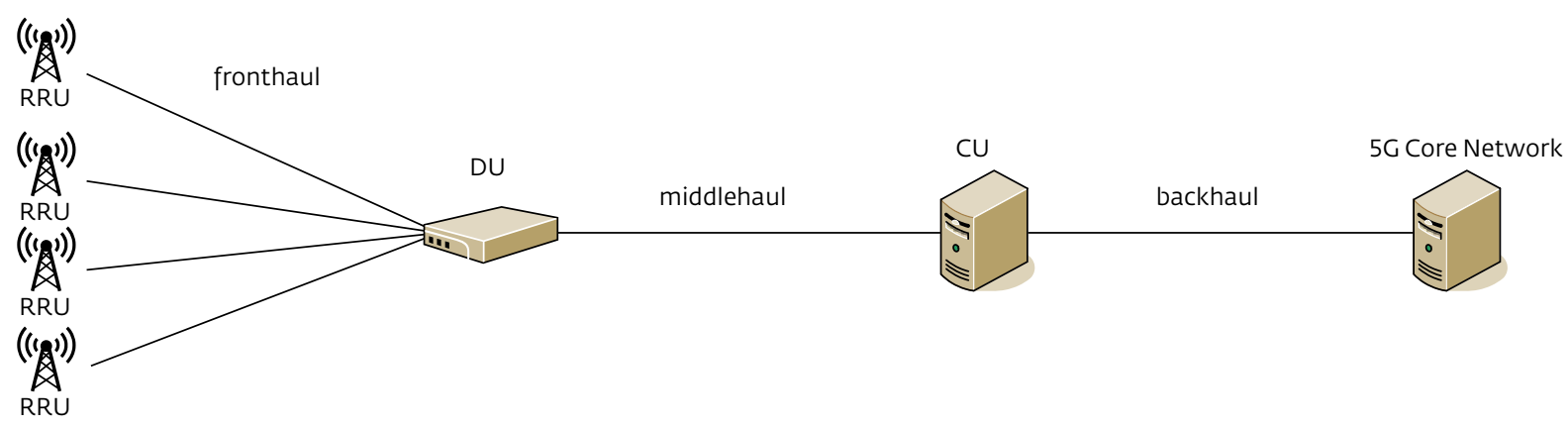

Рис.1. Топология транспортной сети 5G/IMT-2020 (RRU - Radio Remote Unit, DU - Distributed Unit, CU - Central Unit)

требования к полосе пропускания и задержкам предъявляются именно на Fronthaul.

Сегмент, связывающий gNB-DU- и gNB-CUкомпоненты базовой станции, образует Middlehaul. Они могут располагаться физически в одном месте или быть разнесены территориально. CU может подключаться к одному DU или распределять сетевой ресурс между несколькими удаленными модулями. Middlehaul может быть реализован на оборудовании L2/L3. Требования здесь менее жесткие, чем между DU и RRU, так как, в отличие от DU, где имеет место обработка данных реального времени, в центральном модуле CU реализованы только офлайн-алгоритмы.

Транспортное оборудование Backhaul объединяет соты между собой и через метросегмент доставляет их трафик до магистрального ядра сети. Наиболее перспективной технологией Backhaul однозначно остается стек DWDM/OTN, обеспечивающий максимальную пропускную способность и надежность соединений.

Рассмотрим каждый из участков более подробно, и с учетом требований со стороны радиопротоколов определим возможные транспортные интерфейсы.

\section{FronthaUl}

В сетях 4G радиомодуль (RRU) объединяется с антенной и размещается на вершине радиомачты. Базовая станция в основании радиомачты соединяется с радиомодулем оптическим или медным кабелем. Их взаимодействие исторически обеспечивается по протоколу CPRI (англ. CPRI - Common Public Radio Protocol). Теперь, в C-RAN-подходе, радиоинтерфейс должен обеспечить связь DU и RRU на более протяженном участке, так как DU может быть удален на расстояние до 20 км. С учетом большей полосы в радио, агрегации полос, технологии massive MIMO и больших расстояний, разработчики стандарта создали модификацию CPRI - eCPRI. Она отличается эффективным использованием полосы Fronthaul и поддержкой пакетной передачи.

Согласно 3GPPTS 38.401 [2], существует несколько опций функционального разделения между RRU и DU. TaK, CPRI соответствует опции 8, а реализация еCPRI соответствует переходу на опцию 7 (субопции 7.1 и 7.2), где первичная LOWPHY-обработка сигнала осуществляется непосредственно в радиомодуле, снижая требование к пропускной способности и задержке на транспортном уровне (табл.1). Пользовательские данные eCPRI вместе с данными контроля и управления и сигналами синхронизации в виде обычных Ethernet-пакетов передаются по сети, поэтому каждый RRU имеет свой MAC-адрес.

Помимо снижения требований к полосе и, в общем случае, задержки на Fronthaul, преимуществом пакетной передачи является стандартизованная процедура доставки сигналов синхронизации от базовой станции к антеннам (SyncE или 1588v2), а также возможность приоритезации трафика.

Несмотря на все преимущества eCPRI, на первом этапе внедрения сети 5G будут сосуществовать оба поколения мобильной связи (4G/LTE и 5G), а значит, транспортное оборудование должно поддерживать оба протокола.

При построении архитектуры сегмента Fronthaul на физическом уровне (L0) можно выделить несколько групп решений: Point-to-Point (P2P) (т.н. выделенные 
Таблица 1. Требования к транспортному оборудованию Fronthaul для опции 7 и опции 8 функционального разделения в радио

\begin{tabular}{|c|c|c|c|c|}
\hline $\begin{array}{c}\text { Tребование к транс- } \\
\text { портному оборудова- } \\
\text { нию FH }\end{array}$ & \multicolumn{2}{|c|}{$\begin{array}{c}\text { Допустимые значения } \\
\text { при использовании CPRI }\end{array}$} & \multicolumn{2}{|c|}{$\begin{array}{c}\text { Допустимые значения } \\
\text { при использовании } \\
\text { еCPRI }\end{array}$} \\
\hline $\begin{array}{l}\text { Допустимая двусто- } \\
\text { ронняя задержка }\end{array}$ & \multicolumn{2}{|c|}{$\begin{array}{l}\text { От } 50 \text { до } 250 \text { мкс, в зависимости } \\
\text { от мобильного приложения }\end{array}$} & \multicolumn{2}{|c|}{$\begin{array}{l}100 \text { мкс - } 100 \text { мс в зави- } \\
\text { симости от класса } \\
\text { трафика }\end{array}$} \\
\hline $\begin{array}{l}\text { Допустимая ошибка } \\
\text { времени (TE) }\end{array}$ & \multicolumn{2}{|l|}{ $\pm 16 \mathrm{HC}$} & \multicolumn{2}{|c|}{$\begin{array}{l} \pm 10 \text { нс... } \pm 1,36 \text { мкс } \\
\text { в зависимости от класса } \\
\text { трафика }\end{array}$} \\
\hline $\begin{array}{l}\text { Точность установки } \\
\text { частоты }\end{array}$ & \multicolumn{2}{|l|}{$50 \mathrm{ppb}$} & \multicolumn{2}{|l|}{16 ppb } \\
\hline $\begin{array}{l}\text { Точность установки } \\
\text { фазы }\end{array}$ & \multicolumn{2}{|l|}{$1,5 \mathrm{HC}$} & & \\
\hline $\begin{array}{l}\text { Поддержка } \\
\text { интерфейсов }\end{array}$ & $\begin{array}{l}\text { CPRI1 } \\
\text { CPRI2 } \\
\text { CPRI3 } \\
\text { CPRI4 } \\
\text { CPRI5 } \\
\text { CPRI6 } \\
\text { CPRI7 }\end{array}$ & $\begin{array}{l}\text { 614,4 Мбит/с } \\
1228,8 \mathrm{Мбит/с} \\
2457,6 \mathrm{Mбит/с} \\
3072,0 \text { Мбит/с } \\
4915,2 \text { Мбит/с } \\
\text { 6144,0 Мбит/с } \\
9830,4 \text { Мбит/с }\end{array}$ & \multicolumn{2}{|l|}{$\begin{array}{l}10 G E \\
25 G E\end{array}$} \\
\hline \multirow{4}{*}{$\begin{array}{l}\text { Требуемая скорость } \\
\text { передачи данных, } \\
\text { Гбит/с }\end{array}$} & \multirow{4}{*}{ Split 8} & \multirow{2}{*}{ DL 157,3 } & \multirow{2}{*}{ Split 7.1} & DL $37,8-86,1$ \\
\hline & & & & UL 52,8-86,1 \\
\hline & & \multirow{2}{*}{ UL 157,3 } & \multirow{2}{*}{ Split 7.2} & DL 10,1-22,2 \\
\hline & & & & UL 13,6-21,6 \\
\hline
\end{tabular}

существующей инфраструктурой оператора и экономической целесообразностью.

Рассмотрим L1- и L2-уровни модели OSI применительно к Fronthaul. Как часто бывает на сетях операторов, выбор стоит между пакетной и канальной технологиями: Ethernet, подходящий практически для любого протокола L0, и OTN в случае выбора активного WDM на физическом уровне.

Стандартные Ethernetкоммутаторы не могут применяться в сетях 5G, так как им свойственна зависимость задержки от нагрузки, а абсолютное значение может оказаться сопоставимо с требованием на всю сеть нового поколения. Кроме того, при перегрузке сети технология допускает потерю пакетов.

На смену приходит TSN"темные" волокна или "неокрашенные" интерфейсы), WDM в активном или пассивном исполнении, PON (англ. PON - Passive Optical Network) в различных вариантах, а также беспроводной доступ (Micro Wave). Реализация определяется, в частности,

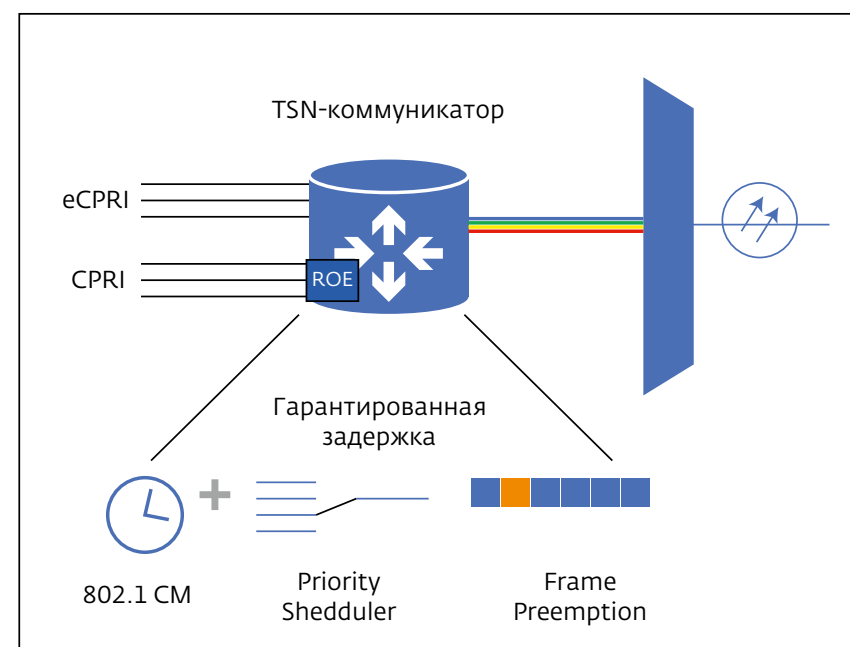

Рис.2. Функции обеспечения предсказуемой гарантированной задержки и роль TSNкоммутатора в сети IMT-2020/5G
Ethernet, который расширяет возможности Ethernet: гарантируется выполнение требований по задержке данного сервисного потока при его передаче по пакетной сервисной сети. Критичные и некритичные к задержкам сервисы могут передаваться совместно. Для этого существуют механизмы, описанные в стандарте IEEE 802.1 CM: Frame Preemption 802.1 Qbu (прерывание на уровне передачи кадров в пользу кадров с большим приоритетом), Enhancements for Scheduled Traffic 802.1 Qbv (управление трафиком, его планирование), Stream Reservation Protocol 802.1 Qcc (SRP - протокол резервирования потока), механизм агрегации и управления ресурсами. Рис. 2 поясняет процедуры, необходимые для передачи сигналов на Fronthaul с помощью расширенного функционала Ethernet-коммутатора.

Существенными недостатками классического Ethernet по сравнению с OTN, помимо вариации задержек, являются: отсутствие встроенных механизмов ОАМ (англ. OАM - Operation, Administration \& Maintenance) на уровне линейного тракта; ненулевая вероятность потери пакетов; отсутствие алгоритма коррекции ошибок. Концепция Ethernet oператорского класса (Carrier Ethernet) предусматривает часть недостающих опций и может быть реализована на обычных Ethernet-коммутаторах. 


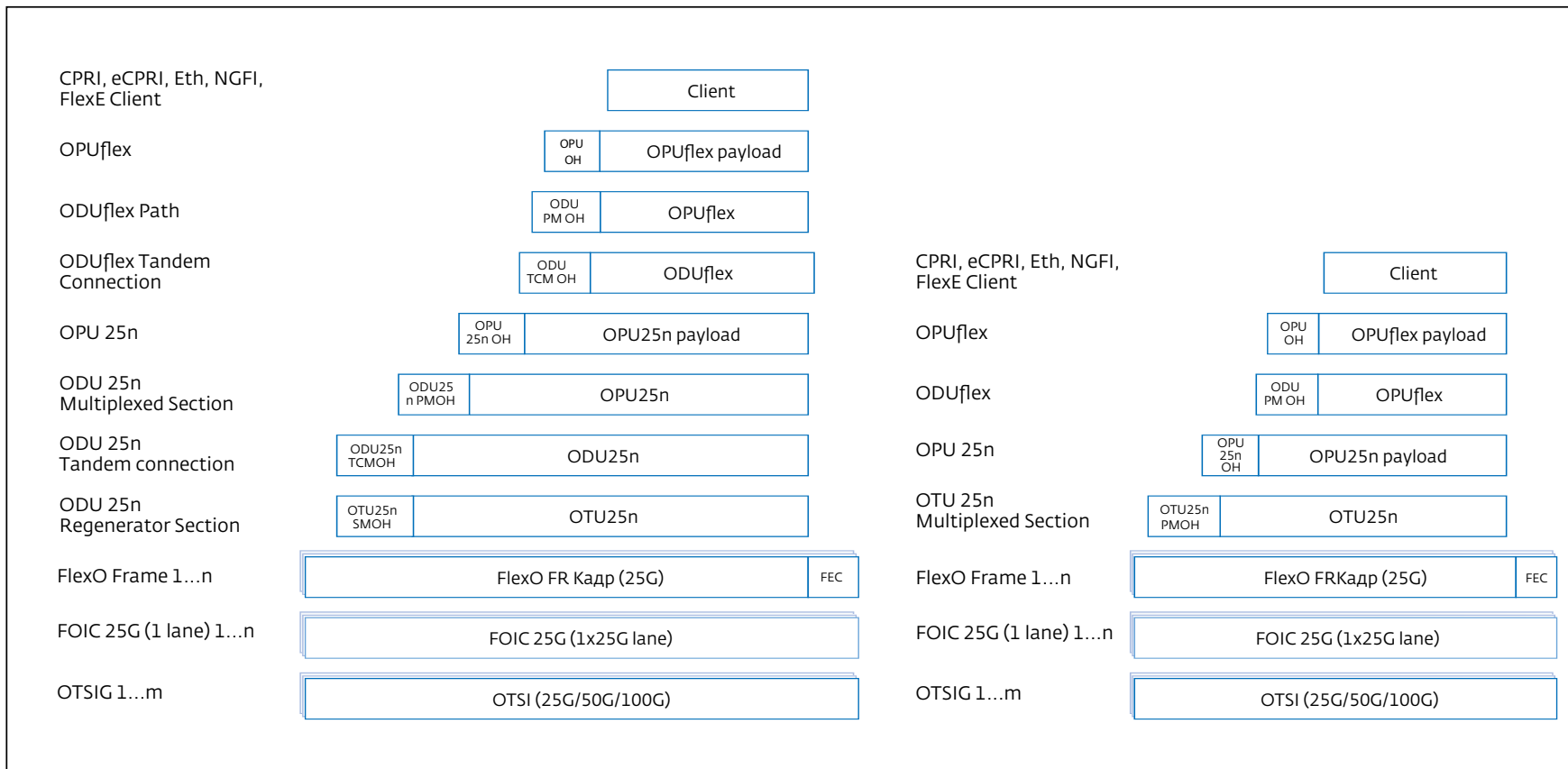

Рис.3. Переход к OTN-lite (инкапсуляция без TCM и SM)

Carrier Ethernet дополнен рядом стандартизован ${ }^{-}$ ных процедур, реализующих масштабируемость, управление сервисами, надежность операторского класса, QoS. Стандарт ITU-TY.1731 определяет процедуры ОАМ в линии, с помощью которых, к примеру, можно определить точное местоположение неисправности в сети.

Стэк OTN/DWDM - это надежность и управляемость, заложенная в идеологии стандарта ITU-TG.709 [3]. Однако не все уровни заголовков целесообразно применять на коротком сегменте Fronthaul в случае выбора технологии. Оптимизация OTN-протокола активно продвигается китайскими вендорами, такими как ZTE, Fiberhome, Huawei. Помимо внедрения на внутреннем рынке, они стараются вывести так называемый OTN-lite на уровень международных стандартов.

Предлагается оптимизировать описанную в стан ${ }^{-}$ дарте ITU-T процедуру инкапсуляции под интерфейсы с битовой скоростью, кратной 25 Гбит/с ("N*25CFlexO", [4]), что позволяет добиться прямой совместимости c eCPRI/Etnernet. Кадр OTU25 после мэппирования и добавления заголовка FЕС формирует кадр 25CFlexO, модулирующий оптическую несущую. Сравнение заголовков для стандартной и оптимизированной процедур инкапсуляции представлено на рис. 3.

В [5] предлагается еще больше сократить число уровней, отображая в FlexO-структуре уже не OTUконтейнер, а непосредственно клиентский сигнал ODUflex. Теперь полезная нагрузка кадра FlexO-n структурируется в виде Р компонентных интервалов (TS), в которых непосредственно отображены ODUflex-клиенты с использованием стан ${ }^{-}$ дартных процедур мэппинга, а служебная нагрузка содержит только заголовки P*TSON и $\mathrm{PMOH}$ для контроля компонентных интервалов и контроля тракта, а также FEC. Предлагается назвать полученную структуру FlexO-FR (рис.4).

CPRI, eCPRI, Eth, NGFI

FlexE Client

Client

\begin{tabular}{|c|c|c|c|}
\hline OPUflex & & $\begin{array}{l}\mathrm{OPU} \\
\mathrm{OH}\end{array}$ & OPUflex payload \\
\hline \multirow[t]{2}{*}{ ODUflex } & & $\begin{array}{l}\mathrm{ODU} \\
\mathrm{PMOH}\end{array}$ & OPUflex \\
\hline & \multicolumn{3}{|r|}{ I or more ODU Clients mapping } \\
\hline FlexOn & $\begin{array}{c}\text { FlexO-n } \\
\mathrm{OH}\end{array}$ & & FlexO-n payload \\
\hline
\end{tabular}

FlexOn Multiplexed section\&FlexOframe $1 \ldots n$

OTSIG

OTSIG $1 . . . \mathrm{m}$

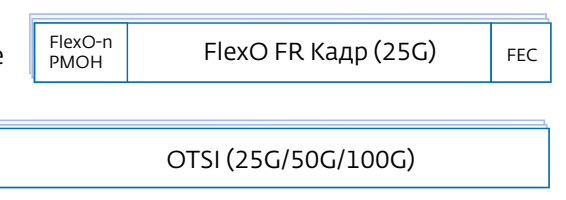

Рис.4. Формирование N"25GFlexO-FR 
Таблица 2. Результаты измерения задержки в оборудовании компании "Т8"

\begin{tabular}{|l|l|l|l|}
\hline \hline \multicolumn{1}{|c|}{ Наименование } & \multicolumn{1}{|c|}{ Режим } & Latency_min, мкс & \multicolumn{1}{c|}{ Latency_max, мкс } \\
\hline \multirow{2}{*}{ MS-100Е-T10 (агрегация 10G в 100G) } & Active & 17,6 & 19,9 \\
\hline \multirow{2}{*}{ T6-10ЕР-01 (транспондер 10G с поддержкой XС) } & G.709 & 23 & 26 \\
\cline { 2 - 5 } & Без FEC & 15,5 & 18,4 \\
\hline \multirow{2}{*}{ ТО-10Р-03 (активный прозрачный WDM) } & Клиент 1GE & $<0,5$ мкс & $<0,5$ мкс \\
\cline { 2 - 5 } & Клиент 10GE & $<0,5$ мкс & $<0,5$ мкс \\
\hline
\end{tabular}

Короткие расстояния и архитектура Fronthaul позволяют отбросить ряд заголовков, характерных для более сложных ОTN-сетей. Заголовки, соответствующие процедурам ТСM (англ. Tandem Connection Monitoring), SM (англ. SectionMonitoring) могут отсутствовать в структуре кадра, что понизит задержку. Обязательным остается только сквозной контроль тракта РМ (англ. Path Monitoting) [5].

Производители оборудования Ethernet и OTN стараются модернизировать оборудование и протоколы, поддерживая сильные стороны конкурирующей технологии с тем, чтобы занять нишу транспорта для Fronthaul. Выбор технологии на участке Fronthaul должен определяться для каждого конкретного операторского сценария. Так, ОTN - это стандартизированные процедуры FEC и ОАМ, возможность организации защитных соединений, процедуры мультиплексирования в высокоскоростные потоки с фиксированной задержкой на уровне единиц-десятков микросекунд. Ethernet - это гибкий протокол с эффективным использованием полосы и новыми процедурами администрирования и минимизации задержек.

Потенциальный сценарий для развертывания ОТN Fronthaul - удаленные от базовой станции модули RRU на расстояние более $10 \mathrm{kM.} \mathrm{Хорошо} \mathrm{ложится} \mathrm{тех-}$ нология OTN на сценарий агрегации потоков CPRI в опции 8-функционального разделения. Такое решение особенно подходит для первого этапа разработки комплексного отечественного решения. Построение Fronthaul на оборудовании Ethernet операторского класса в сочетании с TSN-процедурами, очевидно, подойдет для опций 7.1 и 7.2, в оборудовании RAN, работающем с еCPRI.

В числе технологий физического уровня был также упомянут так называемый активный прозрачный WDM. В отличие от более дорогих транспондеров с OTN-фреймером, формирующим линейный сигнал, "прозрачные транспортные блоки" могут инкапсулировать клиентский сигнал в кадр с фиксированной битовой скоростью, но без сложной иерархии заголовков. Встроенный или внешний оптический мультиплексор позволит эффективно использовать волокно по сравнению, например, c point-to-point-решением, где отдельными волокнами соединяются порты RRU с соответствующими портами DU.

Несмотря на отсутствие ОTN-заголовков, "активный WDM" предусматривает механизмы ОАM: мониторинг канала/линии, индикацию отказов и аварийных состояний в сочетании с минимальной задержкой (табл.2). На таких транспортных модулях может быть организована топология "кольцо" и защитные соединения. Простота реализации позволяет сделать экономичный транспортный блок в уличном исполнении. Преимуществом решения является его универсальность: вне зависимости от опции функционального разделения в радиооборудовании, он может поддержать любой из радиоинтерфейсов (CPRI, eCPRI/ Ethernet, NGFI, OBSAI). Отметим также, что применение подхода "активный WDM" позволяет агрегировать трафик нескольких RRU, на которых отпадает необходимость установки дорогостоящих "цветных" модулей, с большим энергопотреблением и требующих охлаждения.

\section{BACKHAUL}

Интерфейсы. Сегмент Backhaul объединяет трафик базовых станций и транспортирует его к ядру сети. Если выбор транспортной технологии для Fronthaul- неоднозначная задача, где необходимо учитывать множество факторов, то для Backhaul-сегмента только стек DWDM/OTN может обеспечить необходимую пропускную способность в сочетании с надежностью соединений, управляемостью и масштабируемостью. С учетом интерфейсов еNB-DU и eNB-CU важно, чтобы магистральное оборудование имело клиентские интерфейсы 10СЕ, 25СE, 100GE. Эффективное использование ресурсов обеспечивается линейными скоростями 100, 200, 400 Гбит/с в сочетании 
с высокоуровневыми форматами и возможностью программной перестройки. Оборудование ведущих производителей на сегодняшний день удовлетворяет требованиям, предъявляемым к интерфейсам и скоростям передачи.

Задержка. Если в первых коммерческих проектах по внедрению 5G операторы не планируют модернизировать Backhaul-инфраструктуру в предположении, что сеть, построенная с учетом роста трафика 4G, выдержит нагрузку от редких абонентов 5С, то выход второй фазы стандарта может изменить ситуацию.

Модернизация Backhaul может потребоваться операторам при введении 16-го релиза IMT-2020/5G и будет связана с жестким требованием к задержке в URRLCсценарии: суммарная двусторонняя задержка (англ. RTT - Round Trip Time) не должна превосходить 1 мс на всю сеть.

На сегодняшний день типовыми значениями задержек в DWDM считаются единицы-десятки наносекунд для оборудования уровня L0 и единицы-десятки MKC для транспондеров и кросс-коннекта OTN на уровне L1. Фреймеры, используемые в OTN-блоках, обеспечивают существенно меньшую задержку по сравнению с Ethernet/IP (L2/L3), где она достигает единиц и десятков миллисекунд и варьируется в зависимости от загрузки. Наибольший вклад в RTT вносит оптическое волокно, распространение сигнала по которому оценивается величиной 5 мкс/км.

В качестве иллюстрации в табл. 2 приведены результаты измерения задержек в отдельных высокоскоростных блоках производства компании "Т8".

Очевидно, что в дальнейшем при массовом внедрении URLLC-сценария, также как и на Fronthaul, может потребоваться оптимизация структуры кадра OTN [6], уменьшение числа уровней контроля, оптимизация алгоритмов FEC. Так например, принцип "сквозных соединений" в сети 5С допускает отказ от заголовков контроля транспортной секции, оставляя только заголовки мультиплексной секции.

Коммутация. В отличие от архитектуры Fronthaul с наиболее распространенной топологией точка-точка или точка-многоточка между DU и RRU, Backhaul - это сложные mesh-сети. Для распределения ресурсов сети и маршрутизации необходимо предусмотреть механизмы коммутации трафика: в узлах с OTNкросс-коннектом и в мультиплексорах ROADM. Кроме того, необходимая гибкость должна быть обеспечена использованием перестраиваемых по длине волны транспондеров с возможностью изменения формата модуляции в зависимости от запрашиваемого конечными сервисами маршрута.

Требования к коммутационному оборудованию относятся к программной и аппаратной плоскостям.
Рассмотрим пример реконфигурируемого оптического мультиплексора ROADM. Высокоскоростные интерфейсы 200G, 400G и выше спектрально могут представлять собой так называемые суперканалы с несколькими поднесущими, которые могут перенаправляться в различные направления в устройстваx flexROADM.

Гибкость сети обеспечивают так называемые CDCROADM (англ. Colorless, Directionless, Contentionless). Функция Colorless обеспечивает отсутствие привязки длины волны к порту ROADM, то есть возможность вводить и выводить любую длину волны на любом оптическом выходе устройства. Directionless поддерживает составление оптического маршрута от транспондера на любое из направлений ROADM без привязки к входному и выходному портам. Contentionless paзрешает наличие на входных портах сигналов на одинаковых длинах волн, пришедших с разных агрегирующих транспондеров.

Идеология CDC, помимо дорогостоящей аппаратной реализации, должна быть поддержана и на уровне ПO. Так, стандарт Open ROADM MSA (англ. MSA - Multisource agreement) определяет архитектуру ROADM в контексте взаимодействия с верхнеуровневой системой управления. ROADM рассматривается не как отдельное физическое устройство, а как абстрактные цепи управления блоком для общения с системой управления, что делает возможной, в частности, маршрутизацию.

Контроль, управление, маршрутизация. Транспортная инфраструктура - это часть комплексной системы оператора, где все модули должны встраиваться в систему управления. В рамках 5С принято рассматривать три взаимодействующие технологии: SDN (англ. Software Defined Networking), NFV (англ. Network Function Virtualization), ислайсинг (англ. Slicing), образующийся в результате взаимодействия программируемости и виртуализации в сети. SDN обеспечивает программируемость и автоматизацию для обеспечения гибкости и предоставление ресурса по запросу. NFV виртуализирует сетевые функции, что ускоряет ввод новых сервисов и минимизирует задержки в сети. Слайсинг лежит в основе идеологии 5С и организует логические сети поверх физической инфраструктуры с помощью разделения ее ресурсов под потребности разных бизнес-сценариев.

Более подробно остановимся на технологии ASON/ GMPLS, так как она является первым шагом к гибкости и маршрутизации в оптических сетях. При правильной реализации GMPLS-решения на следующем этапе может быть осуществлен мягкий переход к SDN. Отличие заключается в организации плоскости контроля. 


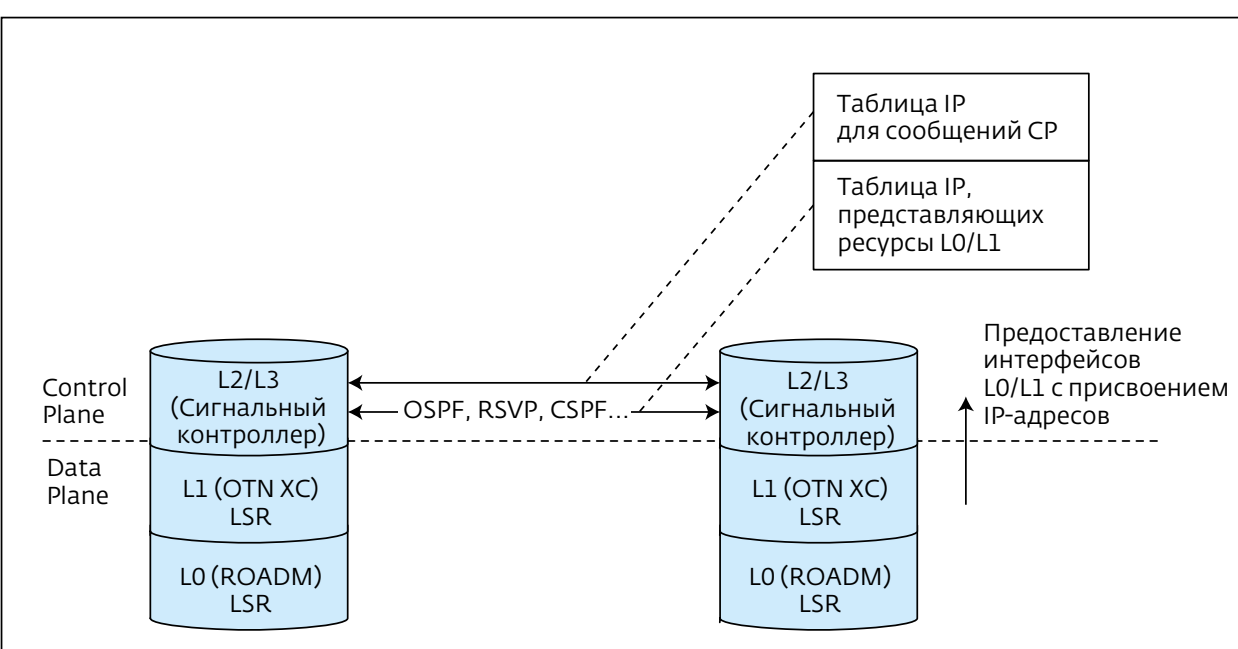

Рис.5. Стек GMPLS-протоколов на модели OSI

контроля и управления приводит к необходимости вводить разную адресацию на этих уровнях.

На уровне плоскости управления действуют стандартные механизмы IP-адресации и маршрутизации, которые обеспечивают доставку сигнализации между сигнальными контроллерами.

Транспортные линки и узлы также должны обладать собственными иден тификаторами, которые необходимо предоставить плоскости управления. В качестве таких идентификаторов физических

Если в SDN верхнеуровневый контроллер собирает информацию и централизованно управляет ресурсами, в GMPLS специальные протоколы распространяют информацию о состоянии сетевых ресурсов между сетевыми элементами, реализуя тем самым "распределенный Control Plane".

Для понимания конкретных механизмов рассмотрим транспортную оптическую сеть как совокупность плоскости данных, плоскости управления и плоскости контроля.

Плоскость данных (англ. Data Plane) включает в себя сетевое оборудование: интерфейсные карты (линейные и клиентские), рассмотренное выше коммутационное оборудование, волоконно-оптическую инфраструктуру.

Уровень управления (англ. Management Plane) может включать в себя систему управления сетевыми элементами EMS (англ. EMS - Element Management System), систему управления сетью NMS (англ. NMS - Network Management System) в зависимости от реализации вендора. Со стороны оператора, использующего инфраструктуру, появляется дополнительная плоскость управления - системы поддержки операций/система поддержки бизнеса OSS/BSS (англ. Operation Support System / Business Support System).

Плоскость контроля (Control Plane) - это слой между плоскостями данных и управления, который вводит интеллект в сетевые элементы в случае ASON/GMPLS или управляет ими "сверху" в SDN.

Так как GMPLS наследует принципы и часть протоколов из MPLS, принцип построения маршрута будет основан на IP-маршрутизации (рис.5). Упомянутое выше разделение плоскости данных и плоскостей устройств (их интерфейсов) также используются IP-адреса. Важно, что IP-контроллеры и IP, ассоциируемые с устройствами плоскости данных, - это два независимых адресных пространства.

В результате в контроллерах плоскости управления формируются таблицы IP-адресов, причем часть из них является IP-адресами контроллеров, по которым происходит обмен сигнальной информацией, а часть отображает топологию низлежащего уровня данных и используется для работы стека протоколов маршрутизации. К ним относится, например, OSPF (англ. Open Shortest Path First), распространяющий информацию о доступных соединениях и реализующий собственно поиск маршрута, протокол сигнализации RSVP (англ. Resource Reservation Protocol) для фиксации заведомо известной длины волны для данного соединения, протокол LMP (англ. Link Management Protocol) - для передачи информации специфичной для кросс-коннекта.

После того, как каждое устройство Control Plane получает всю информацию о топологии плоскости данных, запускается алгоритм расчета известных маршрутов, а лучшие (т.е. маршруты с минимальной метрикой) помещаются в таблицу маршрутизации.

В оптических сетях маршрут может прокладываться с учетом как стандартных критериев (кратчайший путь, минимальная стоимость, минимальное число промежуточных узлов), так и на основе расстояний, потерь в линии, стоимости оптического канала, пропускной способности на данной длине волны, оптического отношения сигнал/шум по дан ному линку и т.д. В сетях 5C для URLLC-приложений особенно важна информация о задержке "из конца 
в конец" - а значит, появляется еще один параметр для расчета пути.

Основное понятие в GMPLS - это так называемый LSP (англ. Label Switch Path), маршрут, формирующийся последовательностью сетевых элементов, поддерживающих коммутацию (ROADM, OTN-XC, транспондеры), и соединяющих их линков, которые используются для доставки трафика между источником и приемником. Сетевые элементы обозначаются английской аббревиатурой LSR (англ. Label Switch Router).

Решение задачи нахождения оптимального маршрута в оптической сети RWA (англ. Routingand Wavelength Assignment) предполагает, что для построения сквозного оптического соединения важно выполнение одновременно трех условий:

- наличие непрерывного оптического маршрута для одной длины волны (Optical Path);

- доступность ресурсов при построении данного маршрута (Resource availability);

- ухудшение качества сигнала из-за оптических эффектов в волокне не должно приводить к увеличению коэффициента ошибок выше допустимого для данного соединения (Impairments Constraints).

В качестве алгоритма нахождения оптимального маршрута классически используется CSPF (англ. Constraint-based Shortest Path First). Для 5С специфичны метрики, то есть критерии, на основе которых выбирается маршрут.

С учетом перспективных требований к мобильному сегменту Backhaul, оптическая метрика может определяться двумя факторами: задержкой (Delay) в оборудовании L0 или L1 и качеством сигнала (в метрике целесообразно использовать величину, обратную оптическому отношению сигнал-шум 1/OSNR).

В сложных mesh-сетях с большим количеством высокоскоростных сигналов в спектре метрика также должна учитывать нелинейные взаимодействия - а значит, правильно оценивать OSNR на каждом участке маршрута, включая его нелинейную составляющую.

В идеальном случае сценарий 5G должен определять метрику с учетом важности того или иного параметра. Если соединение запрашивается еMBВприложением, то маршрут должен быть оптимизирован по оптическому отношению сигнал-шум, критичному параметру для высокоскоростных транспондеров со сложными форматами модуляции. Если ресурс должен быть предоставлен URLLCприложению, метрика должна определяться значениями задержек.

Отметим, что предложенные методы маршрутизации могут использоваться как в ASON/GMPLS-сетях, так и в SDN. Отличие будет заключаться только в уровне вычислений и конкретном элементе, где они будут производиться: в распределенных блоках управления сетевыми элементами или в централизованном контроллере.

\section{ЗАКЛЮЧЕНИЕ}

В статье рассмотрены основные тенденции, которые будут актуальны для разработки линейки транспортного оборудования для мобильных систем пятого поколения - IMT-2020/5G. Описана архитектура решения как в плоскости передачи данных, так и в плоскости управления и администрирования.

В качестве стека технологий для Backhaulи Fronthaul-сегментов рассматривается стек Ethernet/ OTN/DWDM, а также предложены механизмы, адаптирующие отдельные технологии под критичные требования мобильной сети пятого поколения. Отдельно выделена плоскость управления, которая в связке с правильной архитектурой плоскости данных позволяет формировать маршруты для сквозных соединений, запрашиваемых сервисами 5G на основе соответствующих метрик.

Очевидно, что несмотря на зрелость технологий оптического транспорта и соответствующих стан ${ }^{-}$ дартов, модернизация оборудования под сети 5G/IMT2020 неизбежна. Важно в существующий временной зазор между фазами 3СРР-стандартизации совместно с операторами окончательно сформулировать требования к программно-аппаратному комплексу для всех возможных сценариев, а производителям при необходимости доработать существующие линейки транспортного оборудования.

\section{ЛИТЕРАТУРА}

1. Huawei Technologies Co. 5C Transport Networks White Paper: HeavyReadingOperatorSurvey\&Analysis. 2019.

2. 3GPPTS38.401 NG-RAN; Architecture description (Release 15).

3. ITU-T Recomendation G.709 (2016), Interfaces for the optical transport network.

4. ITU-T Recomendation G.709.1 (2017), Flexible OTN short-reach interface.

5. Fiber Home, China Telecommunications Lite and Flexible OTN for next generation (5G) Mobile Fronthaul Transport Lite and Flexible OTN for next generation (5G) Mobile Fronthaul Transport // SG15, C. 0064. Geneva, 2017.

6. Microsemi White Paper The Evolution of ITU-T G.709 Optical Transport Networks (OTN) Beyond 100 Gbit/s. Mar. 2017.

7. Open ROADM MSA White paper Open ROADM overview vol. v1.0. - 2016-10-5. 